\title{
Estudo das características e da hipersensibilidade de lesões cervicais não cariosas em pacientes com alterações oclusais
}

\author{
Study of characteristics and hypersensitivity of non-carious cervical \\ lesions in patients with occlusal changes
}

Viviane Maria Gonçalves de Figueiredo*

Rosenês Lima dos Santos André Ulisses Dantas Batista ${ }^{* * *}$

\section{Resumo}

Objetivo: verificar as características, a extensão e a profundidade da lesão, e a hipersensibilidade das lesões cervicais não cariosas ( $L C N C$ ) em pacientes com alterações oclusais. Sujeitos e método: por meio de um estudo transversal e observacional foram avaliados 58 pacientes com lesões, de ambos os sexos; entre $18 \mathrm{e}$ 71 anos, nos Serviços de Oclusão das cidades de João Pessoa e Campina Grande-PB. Extensão, profundidade e hipersensibilidade de $L C N C$ foram determinadas por exame clínico realizado por único examinador calibrado. A análise dos dados foi realizada por meio de análises descritivas e não paramétricas (coeficiente $\rho$ de Spearman) com 5\% de nível significância e 95\% de intervalo de confiança. Resultados: extensão supragengival com índice de desgaste dental de grau 1 foi prevalente na população estudada. A idade apresentou correlação positiva com o aumento da profundidade das lesões. A hipersensibilidade cervical esteve presente em $74,13 \%$ e mais prevalente nos pré-molares inferiores $(22,34 \%)$. Conclusão: Lesões com extensão supragengival foram prevalentes entre os pacientes da pesquisa; pré-molares inferiores foram o grupo dental mais acometido pela hipersensibilidade e o aumento da profundidade das lesões esteve associado a presença da hipersensibilidade das LCNC.

Palavras-chaves: Epidemiologia. Características. Hipersensibilidade.

\section{Introdução}

As lesões cervicais não cariosas (LCNC) caracterizam-se pela perda da estrutura dentária na região cervical, em nível de junção cemento-esmalte, na ausência de micro-organismos ${ }^{1,2}$ e acometem mais pré-molares ${ }^{3}$. Essas constituem achados rotineiros e cada vez mais comuns na prática da clínica odontológica, a etiologia e classificação são desafiadoras, pois a ação de fatores abrasivos, erosivos e oclusais podem atuar para iniciar a lesão. Assim, a ação desses diverssos fatores causais, determina a etiologia multifatorial dessas lesões ${ }^{4,5}$.

Tais lesões iniciam-se subgengivalmente na região cervical dentária, podem ocorrer em quaisquer das faces dentais e tornam-se mais profundas à medida que é prolongada a ação do seu fator desencadeante ${ }^{6}$. A exposição da superfície dentinária cervical promove a abertura dos túbulos dentinários ao meio bucal, que em presença de um estímulo, promove a movimentação dos fluídos tubulares, atingindo as extremidades dos nervos pulpares e provocando a sensação de dor? Sendo a hipersensibilidade o motivo mais frequente para o paciente buscar ajuda profissional.

Diagnosticar lesões cervicais não cariosas precocemente se faz necessário para prevenir o desenvolvimento de novas lesões, interromper a progressão de lesões instaladas e determinar tratamentos com prognóstico mais favorável ${ }^{8}$. Assim, sabendo 
que pacientes com alterações dos contatos oclusais apresentam um elevado número de $\mathrm{LCNC}^{3}$, se faz necessário e importante investigar outros fatores nesta população como a hipersensibilidade e as características dessas lesões, a fim de fazer com que os profissionais da Odontologia conheçam tais fatores, os quais serão primordiais para traçar a melhor estratégia terapêutica a ser adotada.

Assim, este estudo objetivou verificar as características das LCNC, como extensão, profundidade e hipersensibilidade dessas lesões em pacientes com alterações oclusais. A hipótese a ser testada é que LCNC apresentam correlação com a sensibilidade dentinária.

\section{Sujeitos e método}

Realizou-se um estudo transversal e prospectivo nos Serviços de Oclusão das cidades de João Pessoa-PB e Campina Grande-PB; e a população avaliada correspondeu aos pacientes com 10 pares oclusais que buscaram os serviços em questão, no período de Novembro de 2010 a Outubro de $2011^{3}$. Dentre as alterações oclusais que a população apresentava destaca-se interferências em máxima intercuspidação habitual, alteração da guia lateral e em protusão, e hábitos parafuncionais. Para esta pesquisa, utilizou-se uma amostra por conveniência de 58 pacientes, com base em um estudo anterior realizado nos mesmos serviços ${ }^{3}$, ou seja, apenas pacientes que apresentavam lesões foram avaliados. Foram incluídos no estudo pacientes com pelo menos uma lesão cervical não cariosa e que estivessem dispostos a participar do estudo. Excluiu-se os pacientes com terapêutica dessensibilizante. Para a execução do exame clínico, o examinador e o instrumental (sonda exploradora) haviam sido calibrados anteriormente $^{3}$. A calibração do examinador (calibração intraexaminador) ocorreu durante o exame clínico de pacientes previamente ao estudo; a estatística do Coeficiente Kappa confirmou a reprodutibilidade deste examinador ${ }^{3}$. As sondas exploradoras escolhidas, para o exame clínico, levou-se em consideração a similaridade do diâmetro da ponta ativa ${ }^{3}$. Assim, seguindo a mesma proposta de proporcionar acurácia para os resultados obtidos por meio do exame; as sondas periodontais foram selecionadas quanto à correspondência da milimetragem. $\mathrm{O}$ estudo foi aprovado no comitê de ética local- Hospital Universitário Lauro Wanderley-HULW CAAE 0399.0.126.000-10.

A coleta de dados foi realizada por meio de um exame clínico e formulário específico, quando foram abordadas variáveis sobre as características das lesões como extensão, profundidade e hipersensibilidade.

Extensão da LCNC: classificou-se em ao nível gengival, supragengival e subgengival ${ }^{9}$.

Profundidade da LCNC: o Índice de Desgaste Dental (IDD) definiu a profundidade da perda dentária das lesões em uma escala de 0 a 4 para cada superfície dental avaliada. Os escores desta escala são definidos como: 0 nenhuma alteração de contor- no, 1 mínima perda de contorno, 2 defeito $<1 \mathrm{~mm}$ de profundidade, 3 defeito de 1-2 mm de profundidade e 4 defeito $>2 \mathrm{~mm}$ de profundidade. $\mathrm{O}$ exame para detectar a profundidade das lesões consistiu em posicionar a sonda periodontal milimetrada perpendicularmente ao longo eixo do dente no centro da lesão, identificava-se a profundidade com o escore da escala por meio de uma linha imaginária de continuidade da lesão, de gengival para oclusal ${ }^{10}$.

Hipersensibilidade da LCNC: verificou-se a presença da hipersensibilidade, apenas para as lesões supragengivais, tátil com auxílio de sonda exploradora $n^{\circ} 5$ e evaporativa, utilizando a seringa tripícile a $0,5 \mathrm{~cm}$ da lesão ${ }^{11}$. A distância entre a lesão e a seringa tripícile foi obtida com auxílio de um canudo, inserido na ponta da seringa ${ }^{11}$. A avaliação tátil e evaporativa da hipersensibilidade das lesões foi realizada durante 5 segundos, para cada método de avaliação ${ }^{11}$. A sensibilidade à dor foi quantificada por parâmetros de hipersensibilidade, após cada método de avaliação da hipersensibilidade, questionava-se ao paciente o nível de dor numa escala entre 0 e 3; em que o grau 0 representa sem sensibilidade, grau 1 sensibilidade leve ou suave, grau 2 sensibilidade aguda apenas durante a aplicação do estímulo e o grau 3 sensibilidade persistente após a remoção do estímulo ${ }^{12}$. Previamente a avaliação da sensibilidade da LCNC, foi realizada uma profilaxia nos pacientes com pedra pomes de granulação fina, taça de borracha e caneta de baixa rotação (Dabi Atlante, Ribeirão Preto, São Paulo, Brasil), para eliminar possíveis restos alimentares ou afins que possam apresentar-se sobre as lesão, ocluindo a superfície dos túbulos dentinários e diminuindo a permeabilidade dentinária em cerca de $86 \%$, mascarando a presença ou o grau de sensibilidade da lesão $0^{13}$.

Os dados foram registrados em banco de dados do programa de informática SPSS (Statistical Package for Social Sciences) para Windows, versão 15.0. Adotou-se uma abordagem estatística não paramétrica, pautada na observância da distribuição dos dados, corroborados pelo teste de normalidade de Komogorov-Smirnov, que apontou uma distribuição divergente da normal $(p>0,05)$. Os dados de frequência foram apresentados por meio de tabelas. Os testes realizados foram: U de Mann-Whitney estabeleceu a diferença estatística entre o gênero e o maior número de lesões; Coeficiente $\rho$ de Spearman identificou a correlação entre variáveis métricas, esses resultados foram estabelecidos em gráficos. Adotado um intervalo de confiança de $95 \%$, e nível de significância de $5 \%(\mathrm{p}<0,05)$.

\section{Resultados}

Observou-se média de idade de 33,00 anos, entre 18 e 71 anos; sendo $58,62 \%$ sexo feminino e $41,38 \%$ sexo masculino. A média de dentes com lesões nos gêneros masculino e feminino foi bem semelhante, respectivamente 5,31 e 5,27. Assim, o gênero não esteve, estatisticamente, relacionado ao maior número de lesões ( $p=0,710)$ (Figura 1$)$. 


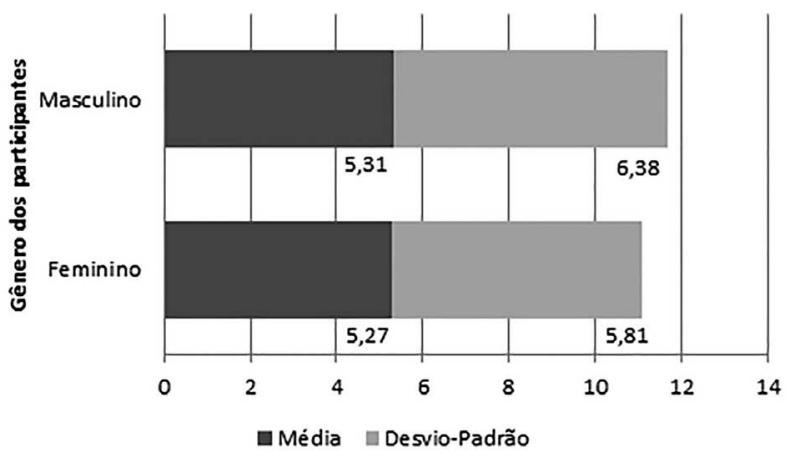

Quanto às características das LCNC, a extensão mais prevalente foi supragengival $(96,4 \%)$, em sequência ao nível $(3,39 \%)$ e subgengival $(0,21 \%)$, bem como repetiu-se esses achados entre os grupos dentais. O IDD grau 1 foi prevalente em todos os grupos dentais (Tabela 1 ).

Figura 1 - Gênero em função do número de dentes com lesões

Tabela 1 - Distribuição das características (porcentagem \%) das lesões cervicais não cariosas por grupos dentários

\begin{tabular}{|c|c|c|c|c|c|c|c|c|c|}
\hline \multirow{2}{*}{ Variáveis } & \multirow{2}{*}{ Grupos } & MS & MI & PMS & PMI & $\mathrm{CS}$ & $\mathrm{Cl}$ & IS & II \\
\hline & & $\%$ & $\%$ & $\%$ & $\%$ & $\%$ & $\%$ & $\%$ & $\%$ \\
\hline \multirow{3}{*}{ Extensão } & Supragengival & 100,00 & 90,24 & 95,74 & 97,32 & 91,11 & 92,31 & 94,64 & 100,00 \\
\hline & Subgengival & 0,00 & 0,00 & 0,00 & 0,00 & 0,00 & 0,00 & 1,79 & 0,00 \\
\hline & Ao Nível & 0,00 & 7,32 & 4,26 & 2,68 & 8,89 & 2,56 & 3,57 & 0,00 \\
\hline \multirow{5}{*}{ IDD } & 0 & 9,09 & 14,63 & 6,38 & 14,29 & 17,78 & 23,08 & 41,07 & 45,00 \\
\hline & 1 & 58,44 & 39,02 & 47,87 & 42,86 & 55,56 & 58,97 & 42,86 & 52,50 \\
\hline & 2 & 23,38 & 26,83 & 35,11 & 32,14 & 13,33 & 15,38 & 14,29 & 2,50 \\
\hline & 3 & 9,09 & 12,20 & 7,45 & 7,14 & 4,44 & 0,00 & 0,00 & 0,00 \\
\hline & 4 & 0,00 & 0,00 & 0,00 & 0,00 & 0,00 & 0,00 & 0,00 & 0,00 \\
\hline
\end{tabular}

${ }^{*}$ MS - molar superior; MI - molar inferior; PMS - pré-molar superior; PMI - pré-molar inferior; CS - canino superior; $\mathrm{Cl}$ - canino inferior; IS - incisivo inferior; II - incisivo inferior.

Correlações estatisticamente significativas foram observadas entre a idade e os escores IDD, ou seja, à medida que os participantes envelhecem, há uma tendência a apresentarem o IDD, nas seguintes intensidades de correlação: fraca para os graus 0 e 3 , moderada para o grau 2 e forte para 0 grau 1 (Grau 0: $\rho=0,353 ; p=0,001$; Grau 1: $\rho=0,663$; p $<0,001$; Grau 2: $\rho=0,524 ; p<0,001$; Grau 3: $\rho=0,234$; $\mathrm{p}=0,028$ ). (Figura 2 )

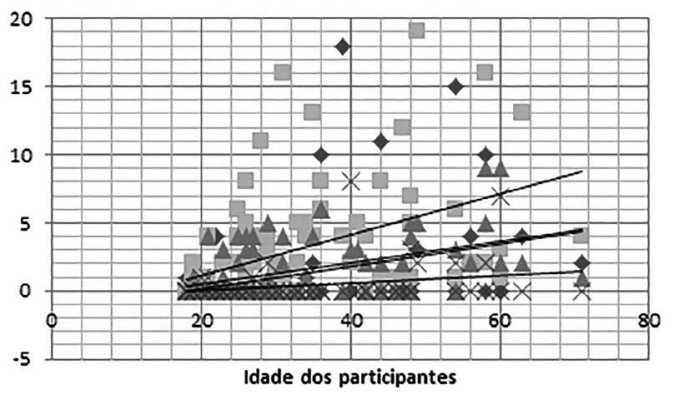

४ IDD Grau 0 IDD Grau 1 $\triangle$ IDD Grau 2 $\times$ IDD Grau 3

Figura 2 - Correlação entre IDD e idade (Teste do coeficiente $\rho$ de Spearman).

A hipersensibilidade dentinária cervical esteve presente em $43(74,13 \%)$ pacientes e em $179(38,82 \%)$ dos elementos acometidos por LCNC, nas seguintes frequências: pré-molares inferiores $(22,34 \%)$, pré-molares superiores $(21,32 \%)$, molares superiores $(14,72 \%)$, incisivos superiores $(9,64 \%)$, caninos su- periores $(8,63 \%)$, caninos inferiores $(5,58 \%)$, molares inferiores $(5,08 \%)$ e incisivos inferiores $(3,55 \%)$. A prevalência da sensibilidade foi maior nos dentes maxilares $(59,78 \%)$ do que nos dentes mandibulares $(40,2 \%)$. As lesões cervicais apresentaram-se mais sensíveis ao estímulo evaporativo (146 lesões) que o estímulo tátil (108 lesões) (Tabela 2).

Tabela 2 - Graus de sensibilidade tátil (ST)* e evaporativa $(S E) *$ em função do grupo dentário**

\begin{tabular}{l|r|r|r|r|r|r}
\hline \multirow{2}{*}{ Grupos } & \multicolumn{1}{c|}{ ST1 } & \multicolumn{1}{c|}{ ST2 } & \multicolumn{1}{c|}{ ST3 } & \multicolumn{1}{c|}{ SE1 } & \multicolumn{1}{c|}{ SE2 } & \multicolumn{1}{c}{ SE3 } \\
\cline { 2 - 7 } & \multicolumn{1}{c|}{$\%$} & \multicolumn{1}{c}{$\%$} & \multicolumn{1}{c}{$\%$} & \multicolumn{1}{c}{$\%$} & \multicolumn{1}{c}{$\%$} & \multicolumn{1}{c}{$\%$} \\
\hline MS & 26,67 & 5,26 & 0,00 & 16,00 & 17,78 & 15,38 \\
MI & 6,67 & 7,89 & 4,00 & 6,67 & 6,67 & 3,85 \\
PMS & 17,78 & 23,68 & 24,00 & 32,00 & 15,56 & 38,46 \\
PMI & 22,22 & 36,84 & 24,00 & 24,00 & 31,11 & 23,08 \\
CS & 6,67 & 13,16 & 12,00 & 4,00 & 8,89 & 7,69 \\
CI & 6,67 & 5,26 & 8,00 & 5,33 & 11,11 & 3,85 \\
IS & 11,11 & 5,26 & 20,00 & 10,67 & 4,44 & 0,00 \\
II & 2,22 & 2,63 & 8,00 & 1,33 & 4,44 & 7,69 \\
Total & 100 & 100 & 100 & 100 & 100 & 100 \\
\hline
\end{tabular}

* ST1 - sensibilidade tátil grau 1; ST2 - sensibilidade tátil grau 2; ST3 - sensibilidade tátil grau 3; SE1 - sensibilidade evaporativa grau 1; SE2 - sensibilidade evaporativa grau 2; SE3 - sensibilidade evaporativa grau 3.

** MS - molar superior; MI - molar inferior; PMS - pré-molar superior; PMI - pré-molar inferior; CS - canino superior; $\mathrm{Cl}$ - canino inferior; IS - incisivo inferior; II - incisivo inferior. 
Os resultados apontaram correlações significativas positivas, de intensidade moderada a forte, entre sensibilidade e IDD nos graus $0 \quad(\rho=0,329$; $p=0,002), 1(\rho=0,730 ; p<0,001), 2(\rho=0,789 ; p<0,001)$, $3(\rho=0,417 ; p<0,001)$ (Figura 3$)$. Optou-se por realizar está análise estatística dentre cada grau de IDD, devido à distribuição dos dados não favorecerem uma análise global da variável; bem como percebesse que é mais importante observar que grau trará está correlação a fim de que o cirurgião-dentista esteja mais atento ao avaliar um paciente com esse tipo de lesão.

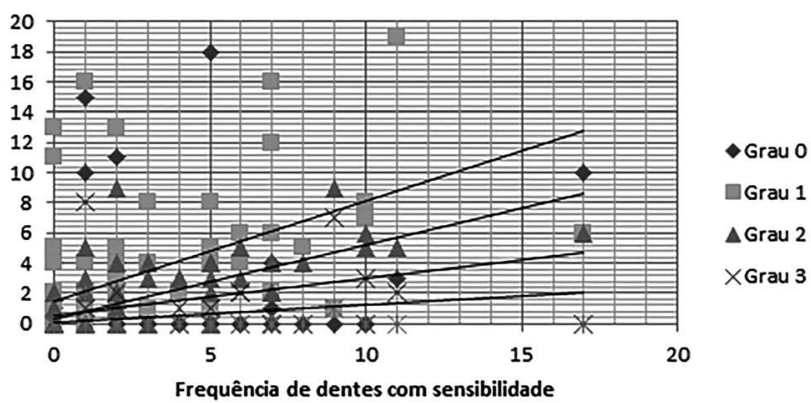

Figura 3 - Correlação entre sensibilidade e IDD (Teste do coeficiente $\rho$ de Spearman).

\section{Discussão}

Estudos clínicos sobre LCNC têm se ampliando devido à elevada frequência dessas lesões na população $0^{3}$. A observação das características como a extensão e o índice de desgaste dentário e da hipersensibilidade são importantes para estruturar o diagnóstico prévio, realizar medidas preventivas e a tomada de decisão para melhor tratamento a ser adotado para tais pacientes.

Confirmando os achados da literatura ${ }^{3}$, o gênero não esteve associado ao maior número de lesões. A localização supragengival tem sido prevalente na literatura ${ }^{9,14}$, alguns estudos detectaram apenas esse tipo de localização $0^{11,15}$, decorrente do critério diagnóstico visual adotado por alguns estudos; embora a sondagem da área cervical com sonda exploradora permita identificar a presença destas lesões "ao prender da sonda" ${ }^{3,20}$. A frequência desse tipo de extensão pode ser explicada pela elevada faixa etária da população em estudo, ou seja, ndivíduos expostos por um longo tempo aos fatores etiológicos das lesões, apresentando um quadro clínico da lesão já instalada. Também se faz interessante observar que, praticamente, todos os grupos dentais apresentaram LCNC ao nível gengival, ou seja, nessa população o número de lesões tenderá a elevar-se, com a ação de fatores etiológicos no decorrer do tempo.

O Índice de Desgaste Dental (IDD) mais frequente nos grupos dentários foi IDD=1 (mínima perda de contorno), sendo que o IDD= 4 não foi observado, enquanto achados na literatura ${ }^{16}$ apresentam IDD prevalente de grau 2 (defeito $<1 \mathrm{~mm}$ de contorno). A diferença de graus no IDD entre o estudo de
Silva ${ }^{16}$ e a pesquisa em questão pode ser explicada pela faixa etária da população do primeiro estudo ser mais elevada em relação ao segundo estudo, a ação de diversos fatores contribuintes a longo prazo interferem para agravar a profundidade da lesão. Os dados apresentaram correlação positiva entre a idade e os graus de IDD, à medida que os indivíduos envelhecem há uma tendência apresentarem maior profundidade da lesão, este resultado também está de acordo com a literatura ${ }^{16}$. A ação de diferentes fatores desencadeantes das lesões promove a progressão dessas ao longo da vida.

Os pré-molares, molares e caninos foram os grupos dentais em que se observou maior frequência de profundidade de desgaste, que pode ser explicado porque tais grupos são os mais acometidos pelas lesões ${ }^{3,11,14,15-20}$ tendem a apresentar lesões mais profundas. Bem como são elementos dentais exigidos nos movimentos excursivos laterais em grupo; assim o longo contato em desoclusão pode deslocar a direção da força e causar lesões atípicas ${ }^{9}$.

O número de LCNC sensíveis foi inferior aos achados da literatura ${ }^{16}$, pois foram avaliadas apenas as lesões supragengivais, sendo que nem todas foram sensíveis; e a prevalência dessas apresentaram IDD=1. Enquanto que o aumento do grau de IDD esteve associado à presença de sensibilidade cervical, ou seja maior profundidade da lesão gera maior exposição de túbulos dentinários, elevando o número de lesões sensíveis ${ }^{11}$. $\mathrm{O}$ presente estudo observou os pré-molares, como os elementos mais acometidos pela sensibilidade cervical, seguidos de molares, incisivos e caninos, conforme a literatura ${ }^{21,22}$. $\mathrm{O}$ arco superior foi o mais acometido pela sensibilidade devido ao fato de ser o arco mais atingido pelas lesões ${ }^{3,19,23}$ e sujeitos à ação de fatores abrasivos e $\operatorname{erosivos}^{20}$.

Nem todas as superfícies dentinárias expostas são sensíveis, a possível explicação para tal afirmação decorre do fato de que os dentes podem se dessensibilizar naturalmente. Proteínas salivares aderem à superfície exterior da dentina e proteínas plasmáticas aderem à superfície interna, bloqueando os túbulos dentinários. Cristais intra-tubulares são formados a partir dos minerais da saliva e do fluido dentinário.

A hipótese a ser testada foi aceita de que lesões cervicais não cariosas apresentam correlação positiva com a sensibilidade dentinária. A limitação deste estudo foi a não aferição dessa sensibilidade após a ação de um desensibilizante e observar como esse vai agir em diferentes profundidades de lesão e faixas etárias.

Os resultados desta pesquisa permitem esclarecer ao cirurgião-dentista o comportamento (características e hipersensibilidade) de lesões cervicais não cariosas em pacientes com alterações oclusais. Partindo deste conhecimento é necessário que a comunidade odontológica olhe para populações acometidas por alterações oclusais e apresentem estra- 
tégias para impedir o avanço das lesões e com esse a hipersensibilidade. Novos estudos clínicos devem ser realizados, na busca de tratar a sensibilidade dentária e traçar a melhor estratégia dessensibilizante, focando o tipo de dentina encontrada em cada lesão a fim de eliminar ou amenizar a dor.

\section{Conclusão}

1. Lesões com extensão supragengival foram prevalentes entre os pacientes da pesquisa;

2. O avanço da idade esteve associado ao aumento da profundidade das lesões;

3. Pré-molares inferiores foram o grupo dental mais acometido pela hipersensibilidade;

4. O aumento do grau do IDD esteve associado à presença da hipersensibilidade das LCNC.

\section{Conflito de Interesses}

Os autores afirmam não haver nenhum conflito de interesse neste estudo.

\section{Abstract}

Objective: to verify the characteristics, length, and depth of the lesion, and the hypersensitivity of non-carious cervical lesions (NCCL) in patients with occlusal changes. Subjects and methods: by means of a cross-sectional observational study, 58 patients of both genders, between 18 and 71 years old, with lesions were evaluated at the Occlusion services of the cities of João Pessoa and Campina Grande, PB, Brazil. Length, depth, and hypersensitivity of NCCL were determined by clinical examination performed by a single calibrated examiner. Data were analyzed using descriptive and non-parametric ( $\rho$ Spearman coefficient) analyses at 5\% significance level and 95\% confidence interval. Results: supragingival extension with grade 1 tooth wear index was prevalent in the population studied. Age was positively correlated with increasing depth of the lesions. Cervical hypersensitivity was present in $74.13 \%$ and more prevalent in lower premolars $(22.34 \%)$. Conclusion: Lesions with supragingival extension were prevalent among patients of the research. The group of lower premolars was mostly affected by dental hypersensitivity, and the increasing depth of lesions was associated with the presence of NCCL hypersensitivity.

Keywords: Epidemiology. Characteristics. Hypersensitivity.

\section{Referências}

1. Michael JA, Townsend GC, Greenwood LF, Kaidonis JA. Abfraction: separating fact from fiction. Australian Dental Journal. 2009 ; $54: 2-8$.

2. Grippo JO, Smiring M, Schreiner S. Attrition, abrasion, corrosion and abnfraction revisited A new perspective on tooth surface lesions. JADA. 2005; 135: 1109-1118.

3. Figueiredo VMG, Santos RL, Batista AUD. Avaliação de hábitos de higiene bucal, hábitos alimentares e pH salivar em pacientes com ausência e presença de lesões cervicais não cariosas. Rer Odontol Unesp 2013; 42(6):414-419.

4. Grippo JO. Abfractions: a new classification of hard tissue lesions of teeth. J Esthet Dent 1991; 3: 14-19.

5. Bartlett DW, Shah P. A Critical Review of Non-carious Cervical (Wear) Lesions and the Role of Abfraction, Erosion, and Abrasion. J Dent Res. 2006; 85: 306-312.

6. Garone Filho W, Silva VA. Lesões não cariosas "O novo desafio as odontologia”. São Paulo: Santos; 2008.

7. Farias GJM, Villela LC. Etiologia e tratamento da hipersensibilidade dentinária em dentes com lesões cervicais não cariosas. Rev Biociênc 2000; 6: 21-27.

8. Sobral MAP, Garone Neto N. Aspectos clínicos da etiologia da hipersensibilidade dentinária cervical. Rev Odontol Univ São Paulo 1999; 13: 189-195.

9. Miller N, Penaud J, Ambrosini P, Bisson-Boutelliez C, Briançon S. Analysis of etiologic factors and periodontal conditions involved with 309 abfractions. J Clin Periodontol 2003; 30: 828-832.

10. Smith BGN, Knight JK. An index for measuring the wer of teeth. J. Br. Dent. 1984; 156: 435-438.

11. Costa LC. Prevalência de lesões dentárias não cariosas e sua relação com o processo erosivo. [Tese de Doutorado]. Bauru: Universidade de São Paulo; 2007.

12. Corona SAM, Nascimento TN, Catirse AB, Lizarelli RF, Dinelli W, Palma-Dibb RG. Clinical evaluation of low-level therapy and fluoride barniz for treating cervical dentinal hypersensitivity. J Oral Rehab 2003; 30(12):1183-1189.

13. Baratiere LN. Odontologia Restauradora Fundamentos e Possibilidades. 6 ed. São Paulo: Santos; 2007.

14. Chan DCN, Browning WD, PohjolaR, Hackman S, Myers ML. Predictor of non-carious lesion of cervical tooth tissues. Operative Debtristry 2006; 31: 84-88.

15. Oliveira ACS, Damascena NP, Souza CS. Análise clínica de pacientes portadores de lesões cervicais não cariosas e sua relação com hábitos. Rev Sul-Bras Odontol 2010; 7: 182-192.

16. Silva FML. Lesões cervicais não cariosas: Prevalência, severidade e correlação com fatores etiológicos. [Dissertação de Mestrado]. Uberlândia: Universidade Federal de Uberlândia; 2006.

17. Bernhardt O, Gesch D, Schawah F, Mack G, Meyer JU, Kocher ET. Epidemiological evaluation of the multifactorial aetiology of abfractions. Journal of Oral Rehabilitation 2006; $33: 17-25$

18. Santos RL, Barbosa RPS, Sales GCF, Costa JDMC. Análise clínica de pacientes portadores de lesões cervicais. Odontologia Clín.-Científ 2005; 4(1):35-42.

19. Young WG, Khan F. Sites of dental erosion are saliva-dependent. Journal of Oral Rehabilitation. 2002; 29: 35-43.

20. Pegoraro LF, Scolaro JM, Conti PC, Telles D, Pegoraro TA. Noncarious cervical lesions in adults Prevalence and occlusal aspects. JADA 2005; 136:1694-1700. 
21. Garcia CH. Avaliação clínica de dois produtos utilizados no tratamento da hipersensibilidade dentinária. [Dissertação de Mestrado]. Rio de Janeiro: Universidade Veiga Almeida; 2005.

22. Aranha ACC. Estudo in vivo da efetividade de diferentes métodos de dessensibilização dentinária em lesões cervicais não cariosas. [Dissertação de Mestrado]. Piracicaba: Universidade Estadual de Campinas; 2003.

23. Piotrowski BT, Gillette WB, Hancock EB. Examining the prevalence and characteristics of abfractionlike cervical lesions in a population of U.S. veterans. J Am Dent Assoc 2001; 132(12):1694-1701.

\section{Endereço para correspondência:}

Viviane Maria Gonçalves de Figueiredo

Praça João Pessoa, n 16, Centro

58297.000 Rio Tinto-PB

Fone 83-88406517

E-mail:vivi_mfigueiredo@yahoo.com.br

Recebido: 31/08/2015. Aceito: 26/03/2017. 